\title{
Safety issues of methylglyoxal and potential scavengers
}

\author{
Shiming LI ${ }^{1}$, Siyu LIU ${ }^{2}$, Chi-Tang HO $(\bowtie)^{2}$ \\ 1 Hubei Key Laboratory for Processing and Application of Catalytic Materials, College of Chemistry and Chemical Engineering, \\ Huanggang Normal University, Huanggang 438000, China \\ 2 Department of Food Science, Rutgers University, New Brunswick, NJ 08901, USA
}

\begin{abstract}
The health safety of methylglyoxal (MGO) has been recognized as a key issue owing to its ultra-high reactivity toward some key biomolecules such as amino acids, proteins, DNA, sulfhydryl- and basic nitrogencontaining compounds, including amino-bearing neurotransmitters. In this review, we have summarized the endoand exogenous sources of MGO and its accumulation inside the body due to high intake, abnormal glucose metabolism and or malfunctioning glyoxalases, and review the debate concerning the adverse functionality of MGO ingested from foods. Higher than normal concentrations of MGO in the circulatory system and tissues have been found to be closely associated with the production of advanced glycation end products (AGEs), increased oxidative stress, elevated inflammation and RAGE (AGE receptors) activity, which subsequently progresses to a pathological stage of human health, such as diabetes complications, cancer, cardiovascular and degenerative diseases. Having illustrated the mechanisms of MGO trapping in vivo, we advocate the development of efficient and efficacious MGO scavengers, either assisting or enhancing the activity of endogenous glyoxalases to facilitate MGO removal, or providing phytochemicals and functional foods containing them, or pharmaceuticals to irreversibly bind MGO and thus form MGO-complexes that are cleared from the body.
\end{abstract}

Keywords reactive carbonyl species, advanced glycation end products, diabetes, brain health, methylglyoxal trapping agents

\section{Introduction}

Methylglyoxal (MGO), one of the dominant reactive carbonyl species (RCS), can be generated both in vitro and in vitro. Exogenously, particularly from the Maillard

Received July 23, 2017; accepted October 12, 2017

Correspondence: ctho@sebs.rutgers.edu reaction during food preparation and processing, $\mathrm{MGO}$ can be derived from Schiff's base and Amadori compounds $^{[1]}$. Therefore, MGO can commonly be detected in various foods or beverages. Significant concentrations of MGO can be found in high fructose corn syrup and manuka honey from New Zealand ${ }^{[2-6]}$. On the other hand, endogenously, MGO is mainly generated from the glycolysis pathway. It can be spontaneously formed from glyceraldehyde-3-phosphate (G3P) and dihydroxyacetone phosphate (DHAP) ${ }^{[7,8]}$.

MGO, also known as 2-oxopropanal, is a highly reactive carbonyl compound. The carbonyl groups of MGO can actively react with amine groups from amino acids, proteins or even DNAs to form the advanced glycation end products (AGEs) and lead to carbonyl stress as well as oxidative stress or even tissue damage. MGO also plays an important role in the formation of color and flavor of foods from the Maillard reaction ${ }^{[1]}$.

\section{Reactive carbonyl species}

Reactive carbonyl species (RCS) are a class of byproducts or intermediates from both exogenous and endogenous oxidation. In general, nucleophilic compounds such as amino acids or proteins can be attacked by RCS covalently and then form harmful adducts. These harmful adducts are named as advanced lipoxidation end products (ALEs) or advanced glycation end products (AGEs) depending on the original source of attacking RCS- from lipids or sugars, respectively ${ }^{[9]}$. The adverse pathophysiological effects which can be induced by the accumulation of RCS compounds as well as the formation of ALEs and AGEs have been described as carbonyl stress in the human body ${ }^{[10]}$. Furthermore, RCS can be divided into three different classes based on their different chemical structures (Table 1): (1) $\alpha, \beta$-unsaturated aldehydes (e.g., 4-hydroxy-trans-2-nonenal and acrolein); (2) di-aldehydes (e.g., glyoxal and malondialdehyde); (3) keto-aldehydes (e.g., MGO and 4-oxo-trans-2-nonenal) ${ }^{[11]}$. 
Table 1 classification and structures of RCS

\begin{tabular}{|c|c|c|}
\hline Classication & Name & Structure \\
\hline \multirow[t]{2}{*}{$\alpha, \beta$-Unsaturated aldehydes } & Acrolein & \\
\hline & 4-Hydroxy-trans-2-nonenal & \\
\hline \multirow[t]{2}{*}{ Di-aldehydes } & Malondialdehyde & \\
\hline & Glyoxal & \\
\hline \multirow[t]{2}{*}{ Keto-aldehydes } & MGO & \\
\hline & 4-Oxo-trans-2-nonenal & \\
\hline
\end{tabular}

\section{MGO generation in vitro}

Exogenously, MGO are mainly generated from the Maillard reaction and sugar autoxidation. In other words, MGO can be formed from the degradation of monosaccharides (e.g., glucose) and Schiff base adducts.

The beginning of monosaccharide autoxidation is the formation of ene-diol. This is caused by deprotonation of carbon-2 of glucose and then the re-distribution of electron density occurs between carbon- 1 and carbon- 2 or carbon- 2 and carbon-3, respectively. Thus, glucose can be transformed to 1,2-enol or 2,3-enol and following the formation of 1-deoxyglucosone (1-DG) or 3-deoxyglucosone $(3-D G)^{[12]}$. MGO can be yielded from the fragmentation of $3-\mathrm{DG}^{[1]}$ (Fig. 1).

MGO can also be generated from the Maillard reaction and the pathway is similar to glucose degradation but in the presence of aldimine can be hydrolyzed into MGO directly $^{[1]}$. The condensation of the carbonyl group from the reducing sugar with the amine group will lead to the formation of Schiff base or aldimine. After rearrangement, the Schiff base can be transformed to fructosamine or 3-DG. Both fructosamine and 3-DG can be degraded into $\mathrm{MGO}^{[13]}$ (Fig. 2).

MGO can be generated from the Maillard reaction at an early stage and it plays important roles in color formation and flavor generation, especially for aroma formation. For example, MGO can be transformed into 1-hydroxy-2propanone through the Cannizzaro reaction, which can be recombined with another MGO to generate 2,5-dimethyl4-hydroxy-3(2H)-furanone (DMHF, furaneol). DMHF is a compound with intense caramel-like aroma as well as a key aroma component generated from the Maillard reaction in many processed foods. Therefore, as a flavor intermediate in the Maillard reaction, MGO can recombine or react with many other carbonyl compounds or amino acids to generate various flavor compounds, such as pyridines, pyrrolines, thiazoles, thiazolines, alkypyrazines, oxazoles and oxazolines ${ }^{[1]}$.

\section{MGO metabolism in vivo}

MGO is ubiquitous in our body because it is a metabolic intermediate and can be generated from the glycolysis bypass $^{[14]}$ (Fig. 3). The major pathway for MGO generation is non-enzymatic and/or enzymatic phosphate
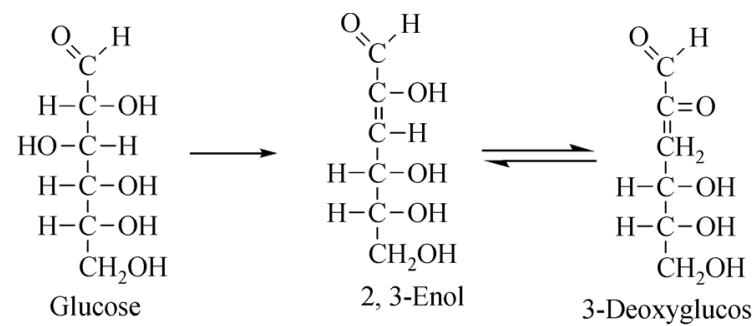

$\mid \begin{aligned} & \text { Retroaldol } \\ & \text { condensation }\end{aligned}$

3-Deoxyglucosone

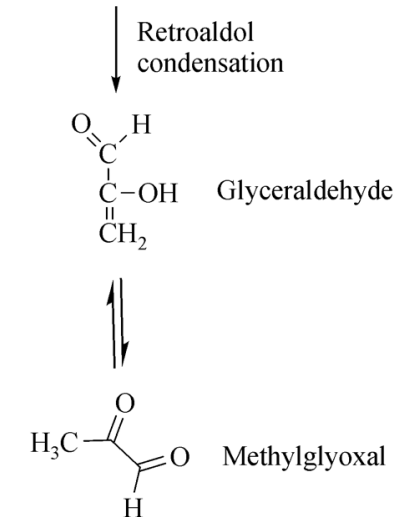

Fig. 1 Oxidative formation of MGO from glucose 


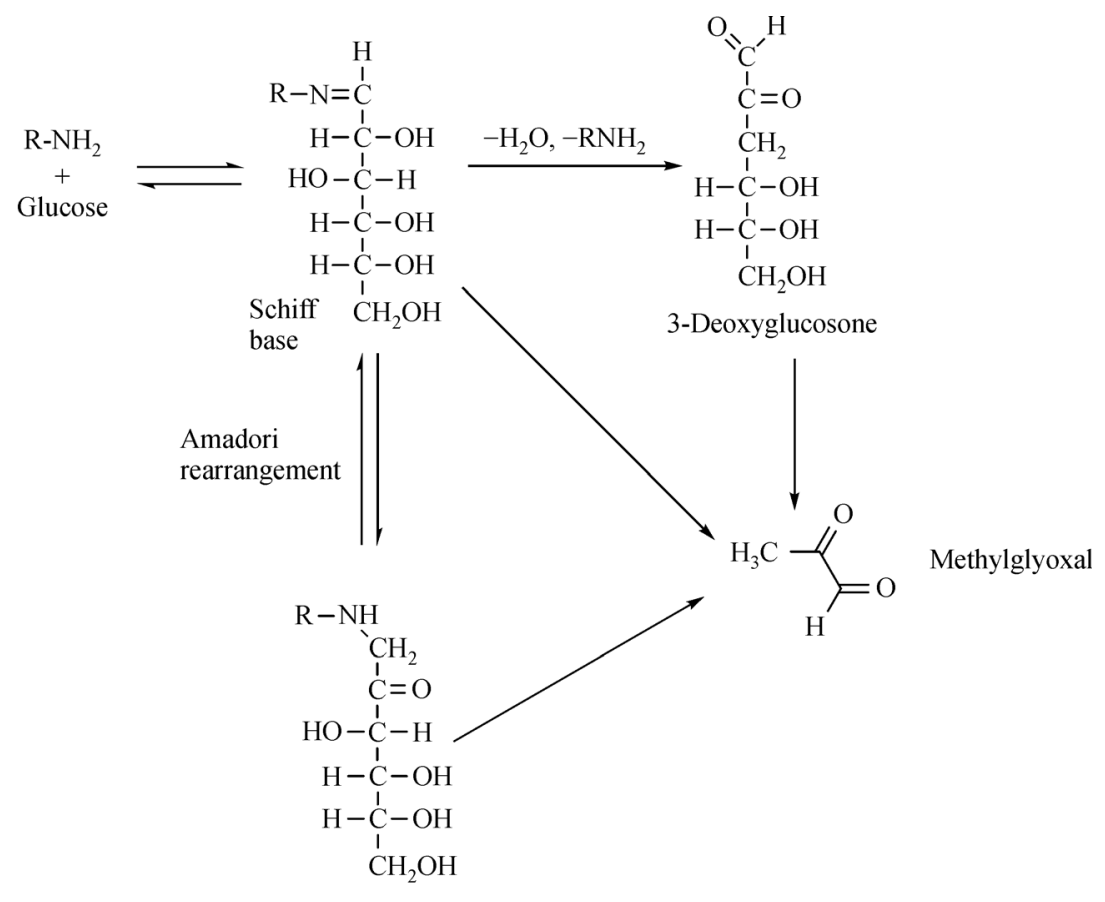

Fig. 2 Formation of MGO in the Maillard reaction

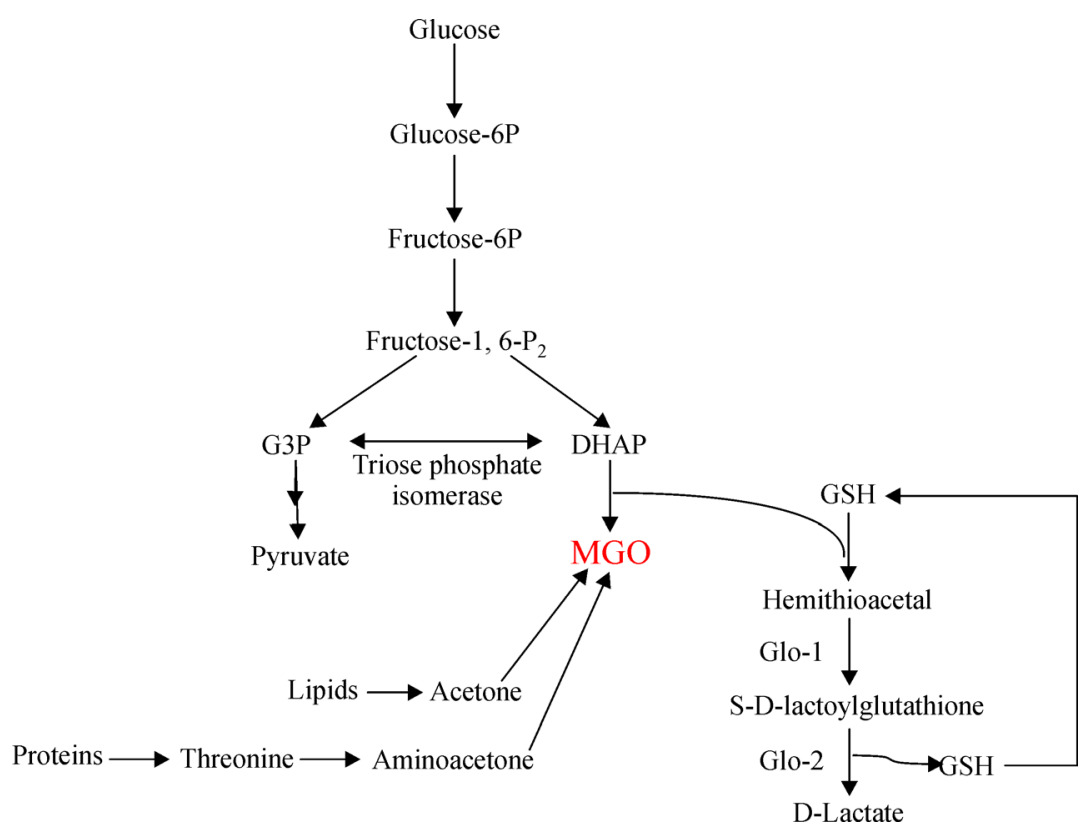

Fig. 3 Metabolism of MGO in vivo

elimination from glyceraldehyde 3-phosphate (G3P) and dihydroxyacetone phosphate (DHAP), via the phosphate enediolate intermediate. Besides glycolysis, there is a small amount of MGO metabolized from fats or proteins in vivo via acetone, threonine or aminoacetone as intermediates ${ }^{[8,15]}$. Although MGO has been confirmed as a cytotoxic compound, its formation still has a controversial role because the glycolysis bypass pathway may have regulatory effects. Normally, G3P will be transformed to pyruvate by the enzyme glyceraldehyde 3-phosphate dehydrogenase (GAPDH) but the increased cellular uptake of carbohydrate compounds such as glucose, or the abnormal glucose metabolism in diabetes, will lead to the inhibition of the GAPDH enzyme, NADH abundance 
and phosphate starvation. As a consequence, the MGO pathway will be activated to relieve the glycolytic burden ${ }^{[8,14,16-18]}$. In this case, the triose phosphate isomerase will catalyze the transformation of G3P to DHAP, synthesizing $\mathrm{MGO}^{[8]}$.

The synthesis of MGO in vivo is inevitable either under healthy or pathophysiological conditions. However, many studies have proved the high toxicity of MGO in cells. Among many different detoxifying pathways, the glyoxalase system is the most important as the major detoxifying mechanism of MGO or other reactive dicarbonyl compounds in vivo to protect our cells from glycation or other oxidative stress. Two enzymes, glyoxalase-1 (Glo-1) and glyoxalase-2 (Glo-2) and one additional necessary cofactor glutathione (GSH) are functioning coherently in the biological system. At first, GSH can react with MGO and yield hemithioacetal. Then, Glo-1 will catalyze hemithioacetal to S-D-lactoylglutathione. Subsequently, under the catalysis of Glo-2, S-D-lactoylglutathione will be transformed to D-lactate, which can be excreted into urine easily. Simultaneously, GSH will be recycled back to the system (Fig. 3). But a high concentration of MGO in the system comparing normal conditions or the inefficiency of Glo-2, may lead to the depletion of free GSH and the inability to recycle in the system. As a result, this will lead to greater accumulation of MGO in cells, which will induce glycation, increase oxidative stress and promote the development of many degenerative conditions such as diabetic complications, cardiovascular disease and Alzheimer's disease ${ }^{[19]}$.

\section{$5 \quad$ MGO and AGEs}

MGO is a typical RCS compound which can lead to the AGEs formation under physiological conditions. Due to its high reactivity and ubiquitous generation, MGO has been regarded as the most important source of AGEs. Compared with glucose in glycation reactions, MGO can be as much as 20000 times more reactive ${ }^{[20]}$. In particular, MGO reacts with arginine residues, regarded as the most active glycation. Other reactions, occurring to a lesser extent than arginine, are with lysine, cysteine and tryptophan ${ }^{[21]}$. The reaction schemes are shown in Fig. 4.

The reaction between MGO and arginine will lead to the formation of cyclic imidazolone adducts. Depending on the nitrogen atoms involved in the cyclization as well as the different environmental $\mathrm{pH}$ value, there are three different structural isomers formed: $\mathrm{N}^{\mathrm{d}}-(5$-methyl-4-imidazolon-2-yl)-L-ornithine ( $M G$ - $H 1)$, 2-amino-5-(2-amino5-hydro-5-methyl-4-imidazolon-1-yl) pentanoic acid ( $M G$ H2) and 2-amino-5-(2-amino-4-hydro-4-methyl-5-imidazolon-1-yl) pentanoic acid $(M G-H 3)$. These three isomers are adducted in equilibrium. Because they can open and give the carboxyethylarginine (CEA) adduct as well as reverse back to re-cyclize, mutual interconversion can

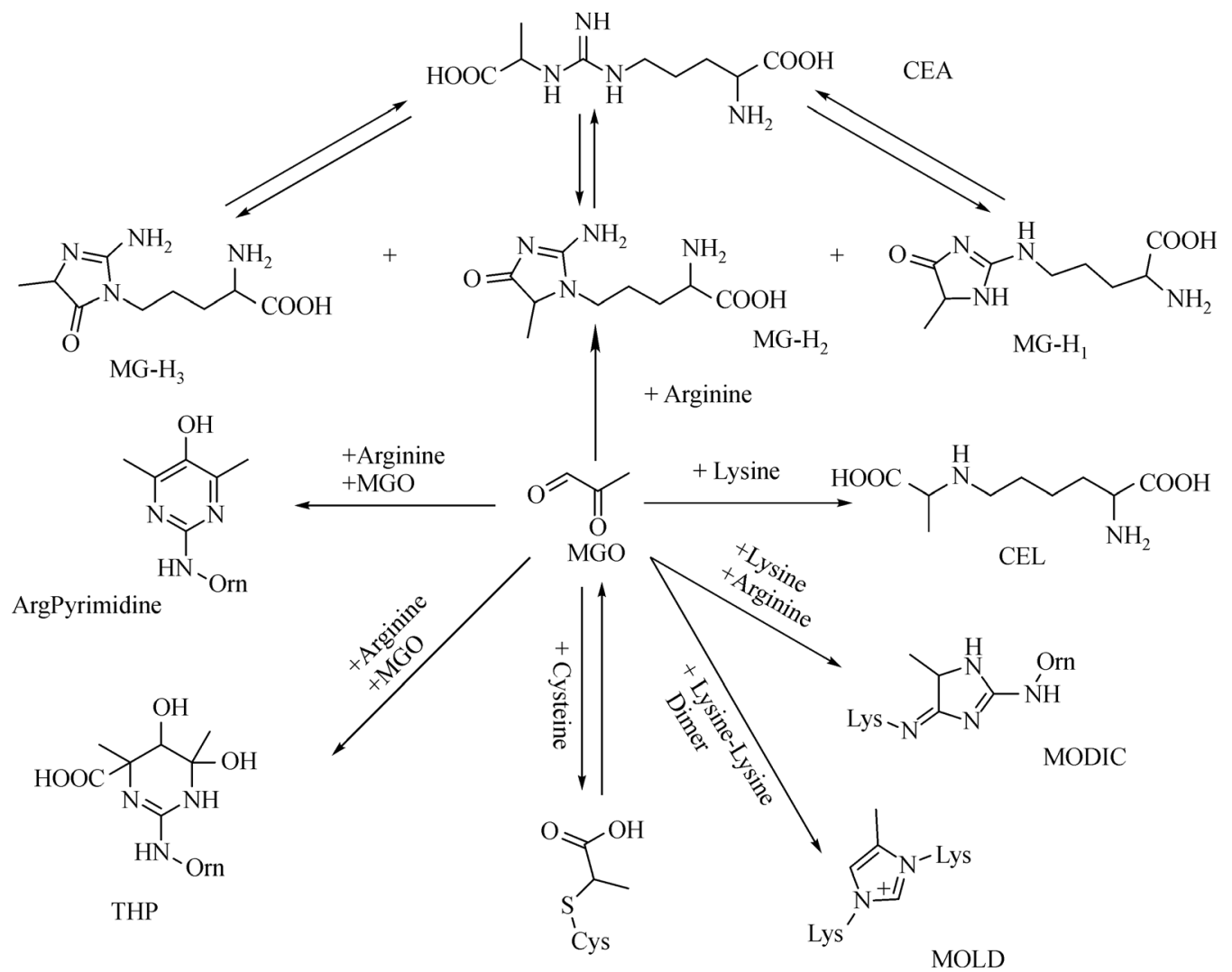

Fig. 4 Major pathways for the formation of MGO-derived AGEs 
occur between the three isomer adducts. Furthermore, an additional methylglyoxal can be added and yield either THP $\left(\mathrm{N}^{\mathrm{d}}\right.$-(4-carboxy-4,6-dimethyl-5,6-dihydroxy-1,4,5,6tetrahydropyrimidine-2-yl)-L-ornithine) or argpyrimidine $\left(\mathrm{N}^{\mathrm{d}}-(5-\right.$ hydroxy-4,6-dimethylpyrimidine-2-yl)-1ornithine $)^{[21-25]}$.

MGO can react with lysine residues to form a carboxylethyllysine (CEL) adduct with the aldimine as an intermediate ${ }^{[26]}$. With the lysine dimer, MGO can be adducted to MOLD (6-\{1-[(5S)-5-ammonio-6-oxido-6oxohexyl]-4-methyl- imidazolium-3-yl\}-L-norleucine). With one lysine and one arginine residue, MGO can form MODIC adduct (2-ammonio-6-(\{2-[4-ammonio-5oxido-5-oxopentyl) amino]-4-methyl-4,5-dihydro-1H-imidazol-5-ylidene $\}$ amino)hexanoate) ${ }^{[27,28]}$. Additionally, MGO can also react with cysteine residues to form reversible hemithioacetal adducts, and with tryptophan residues can generate $\beta$-carboline derivatives ${ }^{[29,30]}$.

Because of the abnormal glycation or crosslinkage, amino acids and proteins will be dysfunctional and lead to inflammation, oxidative stress, tissue injury or cell apoptosis $^{[31]}$. As a result, the MGO-derived AGEs have been associated with the development of many degenerative healthy conditions, such as cataracts, cardiovascular disease, diabetic complications, nephrosis or brain health problems ${ }^{[32]}$. For example, significant increases of CML in cerebrospinal fluid has been detected in amyotrophic lateral sclerosis patients ${ }^{[33]}$ and CML level in cortical neurons and cerebral vessels has been reported to affect the severity of cognitive impairments among people with cerebrovascular diseases ${ }^{[34]}$.

\section{MGO and oxidative stress}

MGO is regarded as a toxic compound because not only can MGO itself increase oxidative stress but also the AGEs formed have the potential to lead to elevation of oxidative stress $^{[35]}$. Studies has shown that oxidative stress for healthy rats can be increased significantly with oral consumption of $\mathrm{MGO}^{[36]}$. Many other researchers have revealed MGO-induced oxidative stress pathways. For example, MGO can increase the formation of superoxide, hydrogen peroxide, peroxynitrite and proinflammatory cytokines in different cell types such as vascular smooth muscle cells, rat hepatocytes, neutrophils, and platelets among others ${ }^{[37-40]}$. MGO can also promote the activity of several pro-oxidant enzymes such as NADPH oxidase ${ }^{[37]}$. Additionally, MGO can amplify the oxidative stress by reducing several antioxidants in vivo such as GSH, glutathione peroxidase and glutathione reductase ${ }^{[41,42]}$.

Furthermore, the formation of AGEs will not only affect the function of proteins and DNAs, it will also activate the membrane receptors, typically RAGE (receptors of AGEs), which can trigger specific intracellular signals ${ }^{[35]}$. RAGE is water soluble and has been proposed as the most important receptor for AGEs because it can recognize two major types of AGEs, CML adducts and imidazolones, through specific regions and then activate NF- $\mathrm{KB}^{[31]}$. As a result, elevation of oxidative stress will occur and subsequently provoke positive inflammatory feedback, apoptosis, macrophage, platelet activation, thrombosis or cause the progression of vascular complications ${ }^{[8]}$.

\section{$7 \quad$ MGO and type-2 diabetes}

As previously mentioned, the increased consumption of carbohydrates, like glucose, or abnormal glucose metabolism in diabetes will activate the MGO pathway in glycolysis and generate more MGO compared with normal conditions. Therefore, the MGO concentration in diabetic patients' body will be 2-6 times higher than healthy people $^{[43]}$. This can be one of the reasons why diabetic patients normally suffer with diverse complications, such as cataracts, cardiovascular disease, nephrosis or neuro and brain health problems ${ }^{[7]}$.

On the other hand, an overwhelming amount of MGO in vivo will also induce type-2 diabetes or its complications. Insulin resistance and $\beta$-cell loss are the hallmarks of type2 diabetes ${ }^{[8]}$. Many studies have focused on the pathological effects of MGO on diabetes and the major effect is related to insulin. Insulin is the peptide hormone produced from pancreatic $\beta$-cells regulating glucose homeostasis. The highly reactive carbonyl compound MGO can react with the $\mathrm{N}$ terminus and arginine residue of human insulin. The formation of MGO-insulin adducts will affect insulinmediated glucose uptake, impair autocrine control of insulin release from $\beta$-cells and decrease hepatic clearance of insulin from liver cells ${ }^{[44,45]}$. The glycation of insulin will also damage the ability of insulin to bind or activate its receptor and probably lead to insulin resistance ${ }^{[35]}$. Other possible pathological effects of MGO with type- 2 diabetes include effects on glucose transporter, pancreatic $\beta$-cell anion channel, hemogloblin, and endothelial cells among others $^{[8]}$.

\section{MGO and brain health}

MGO can be generated spontaneously and continuously in all mammalian cells. Due to its high reactivity and oxidative ability, the accumulated MGO can attack proteins and DNAs or other biomolecules to form AGEs and cause the irreversible serious loss of function or damage to organs ${ }^{[46]}$. However, on the other hand, brain has the high energy needs and glucose is the major energy source for the brain. As a by-product of glycolysis, MGO generation and accumulation will be increased as the glycolysis rate elevated ${ }^{[19]}$. Therefore, it is not hard to understand that the amount of MGO in cerebrospinal fluid (CSF) will be five to seven times higher than in plasma. 
Briefly, MGO itself may have adverse effects on neurons directly because of depolarization, ROS production or as a result of cell apoptosis ${ }^{[47]}$. What is more, glycation or carbonyl stress have been involved in neurological and neurodegenerative disorders ${ }^{[48]}$. For example, MGO may promote abnormal protein aggregation due to crosslinkage and induce oxidative stress. Recently, the relationship between MGO and brain health problems is attracting more attention.

The role of MGO in Alzheimer's disease (AD) has been studied widely. The studies revealed the higher concentration of MGO in CSF of diabetic patients, the pathogenesis of $\mathrm{AD}$ might be accelerated. Extracellular amyloid- $\beta(\mathrm{A} \beta)$ and intracellular neurofibrillary tangles (NFTs) are two distinct features of $\mathrm{AD}$. These two protein aggregations are more stable and long-lived compared with other normal proteins, thus they are more readily glycated. After glycation, the AGE adducts, for example $\beta$-amyloid plaques, will be more insoluble and protease-resistance ${ }^{[21]}$. Another study has shown that the quantity of AGE adducts in $\mathrm{AD}$ brains are three times higher than in healthy brain, and the AGE accumulation may also promote the aggregation of additional amyloids ${ }^{[49]}$. Additionally, the A $\beta$ AGEs can also be recognized by RAGE. As a result, the oxidative stress, inflammation, amyloidosis and the neurotoxicity will be increased for microglia, the blood brain barrier and neurons ${ }^{[50,51]}$.

There are also many studies focused on glycation and amyotrophic lateral sclerosis (ALS) and the results showed that the copper-zinc enzyme superoxide dismutase (SOD1), which can catalyze the quenching of superoxide radicals, is susceptible to be glycated because of several lysine and arginine residues in the primary structure of the enzyme. CML and non-CML AGEs are found in the anterior horn motor neurons and microglia in the spinal cord of ALS patients ${ }^{[48,52,53]}$. Data showed there is no increase of AGEs in multiple sclerosis (MS) patients compared with healthy people, but RAGE receptors in MS patients and animal models has been upregulated ${ }^{[48,54,55]}$.

In this century, MGO and its glycation have been suspected to be involved with Parkinson's disease (PD). Similar to $\mathrm{A} \beta$ and NFTs for $\mathrm{AD}$, Lewy bodies are hallmarks in PD pathology. Studies showed the Lewy bodies can be modified to AGEs which then alter RAGE expression $^{[48,56,57]}$, which may lead to dopaminergic neurons destruction.

\section{MGO trapping agents}

The accumulation of MGO in cells will lead to carbonyl stress and AGEs formation or even induce degenerative health conditions especially for diabetes mellitus. Until now, there are several available pharmaceutical AGE inhibitors that can be used to trap MGO or prevent AGE formation and relief the diabetic complications. One example is aminoguanidine, a nucleophilic hydrazine compound which can react with MGO rapidly and inhibit AGEs formation and other related degenerative complications $^{[32,58]}$.

Although pharmaceutical compounds can trap MGO or inhibit AGEs formation significantly, their adverse side effects have limited their clinical usage ${ }^{[7]}$. Many natural phenolic compounds show significant effects in trapping MGO and some flavonoids have shown significant inhibitory effects on AGE formation which is mediated by MGO. For example: luteolin, rutin, (-)-epigallocatechin-3-gallate (EGCG) and quercetin can inhibit $82.2 \%$, $77.7 \%, 69.1 \%$ and $65.5 \%$ AGE formation, respectively ${ }^{[59]}$. From this perspective, some flavonoids have already been shown to trap MGO efficiently and alleviate AGE formation. Under physiological conditions $(\mathrm{pH} 7.4$, $37^{\circ} \mathrm{C}$ ), EGCG from tea can trap over $90 \% \mathrm{MGO}$ in $10 \mathrm{~min}$ and form 8-mono-MGOEGCG, 6-monoMGOEGCG and 6,8-di-MGOEGCG adducts as the products based on the different ratio of MGO and EGCG in the reaction conditions ${ }^{[60]}$. Similarly, genistein from soybean, quercetin from diverse plants and phloretin or phlorizin from apple can also trap MGO effectively and form both mono-MGO adducts or di-MGO adducts based on different reaction reagents under controlled physical conditions $\left(\mathrm{pH} 7.4,37^{\circ} \mathrm{C}\right)$. More than $80 \%$ of MGO can be trapped by genistein within $4 \mathrm{~h}$ and the trapping efficiency can be as high as $97.7 \%$ after $24 \mathrm{~h}^{[61]}$. Quercetin can trap $80.1 \%$ of MGO after $1 \mathrm{~h}^{[62]}$. More than $80 \%$ MGO can be trapped by phloretin within $10 \mathrm{~min}$ and more than $70 \%$ MGO can be trapped by its glucoside phloridzin within $24 \mathrm{~h}^{[63]}$. Although some flavonoids or phenolic compounds has shown significant ability in scavenging MGO, these studies have been mainly focused in vitro. The ability of genistein to trap MGO has been studied in vivo. In vivo MGO trapping condition is much more complicated because of several factors: (1) systemic bioavailability of flavonoids; (2) metabolism and activities of their metabolites; (3) oxygen pressure; (4) $\mathrm{pH}$; (5) presence of other endogenous and exogenous compounds and so on. Based on these results, only mono-MGO adducts of genistein were found. Both mono-MGO genistein adducts and mono-MGO genistein metabolite adducts were found in mice urines. Except for two genistein metabolites, 6hydroxygenistein and 8-hydroxygenistein, all other metabolites of genistein can be adducted by MGO and found in the mice urine samples, showing that genistein and its metabolites which share the same A ring structure can trap $\mathrm{MGO}^{[64]}$.

Besides studying the MGO trapping ability of certain specific flavonoids or phenols, some research has focused on the comparison of different flavonoids in parallel experiments between compounds with similar structures in order to study the MGO scavenging mechanism. One such study focused on 20 different single benzene phenolic structure compounds which were incubated with MGO 
seperately in the ratio of 1:1 under physiological conditions. The conclusion was that mono-hydroxyl and dihydroxyl benzene compounds are not efficient at trapping MGO. Benzenetriols, however, can trap MGO efficiently. The position of a carboxylic group on the benzenetriol structure can play a key role in the reaction ${ }^{[65]}$. Another study was more focused on the flavonoids (quercetin, luteolin, epicatechin, genistein, daidzein, apigenin and phloretin). Five typical phenolic compounds (gallic acid, phloroglucinol, pyrogallol, pyrocatechol, and resorcinol) were investigated in the research to simplify the influential factors. The results showed pyrogallol has the highest MGO-trapping ability with $90 \%$ of MGO trapping in $24 \mathrm{~h}$. The MGO-trapping ability sequence of other compounds investigated is phloroglucinol $(60.5 \%)$, resorcinol (31.6\%), pyrocatechol (21.8\%) and gallic acid (14.9\%). This research revealed that 1,2,3-trihydroxybenzen (pyrogallol) has higher MGO-trapping ability than other 1,2-dihydroxybenzene and 1,3-dihydroxybenzene. Substitution at position 5 of pyrogallol decreased the trapping ability which means the position 5 is the active site of pyrogallol to trap MGO. Comparisons of phloroglucinol and resorcinol comparison as well as genistein (90\%) and daidzein (54.5\%) show that the A ring is crucial for MGO trapping and the hydroxyl group on C-5 in the A ring is very helpful for MGO-trapping abilities of flavonoids. On the other hand, the comparison between quercetin $(90 \%$ per $24 \mathrm{~h}$ ), luteolin and (90\% per $24 \mathrm{~h}$ ) and epicatechin ( $85 \%$ per $24 \mathrm{~h}$ ), suggests that the double bond between $\mathrm{C}-2$ and C-3 can promote the MGO trapping ability of flavonoids. The other conclusion from this study was that the number of hydroxyl groups on B ring does not influence the MGO-trapping abilities significantly for flavonoids ${ }^{[66]}$.

\section{Conclusions}

MGO, one of the reactive carbonyl compounds, has attracted more and more attention because of its potential close relationship with many degenerative healthy problems. MGO can actively react with the amine group of amino compounds and form AGEs as the products. Furthermore, the amount of MGO is about 2-6 times higher in diabetic patients' bodies compared to healthy people, which is one reason why diabetic patients suffer with different complications such as cataracts, cardiovascular disease and nephrosis. In this decade, more and more research studies have focused on the relationship between MGO and brain healthy problems like AD and PD. For the $\mathrm{AD}$, many studies showed the higher concentration of MGO in CSF for diabetic patients may accelerate the AD progress. For example, MGO may actively glycate extracellular amyloid- $\beta(A \beta)$ to $\beta$-amyloid plaques which are more insoluble and protease-resistance. For the PD, the epidemiological studies also showed that diabetic patients are more frequently diagnosed with PD compared with healthy people, which has raised the suspicion that the higher amount of MGO may also increase Parkinson's disease.

Since MGO is a common Maillard reaction intermediate, it exists in daily processed foods. It is still not established whether dietary MGO will cause any safety issue and this remains an urgent issue for food safety. In addition, it is well-known that certain phytochemicals such as flavonoids may trap MGO in vitro, and they may be used to reduce the amount of potential toxic MGO in commonly processed foods.

Acknowledgements This work was supported by the USDA National Institute of Food and Agriculture, Hatch project 1007898.

Compliance with ethics guidelines Shiming Li, Siyu Liu, and Chi-Tang Ho declare that they have no conflicts of interest or financial conflicts to disclose.

This article is a review and does not contain any studies with human or animal subjects performed by any of the authors.

\section{References}

1. Wang Y, Ho C T. Flavour chemistry of methylglyoxal and glyoxal. Chemical Society Reviews, 2012, 41(11): 4140-4149

2. Degen J, Hellwig M, Henle T. 1,2-Dicarbonyl compounds in commonly consumed foods. Journal of Agricultural and Food Chemistry, 2012, 60(28): 7071-7079

3. Revel G D, Pripis-Nicolau L, Barbe J C, Bertrand A. The detection of $\alpha$-dicarbonyl compounds in wine by formation of quinoxaline derivatives. Journal of the Science of Food and Agriculture, 2000, 80(1): 102-108

4. Lo C Y, Li S, Wang Y, Tan D, Pan M H, Sang S, Ho C T. Reactive dicarbonyl compounds and 5-(hydroxymethyl)-2-furfural in carbonated beverages containing high fructose corn syrup. Food Chemistry, 2008, 107(3): 1099-1105

5. Mavric E, Wittmann S, Barth G, Henle T. Identification and quantification of methylglyoxal as the dominant antibacterial constituent of Manuka (Leptospermum scoparium) honeys from New Zealand. Molecular Nutrition \& Food Research, 2008, 52(4): 483-489

6. Hayashi T, Shibamoto T. Analysis of methyl glyoxal in foods and beverages. Journal of Agricultural and Food Chemistry, 1985, 33 (6): 1090-1093

7. Löbner J, Degen J, Henle T. Creatine is a scavenger for methylglyoxal under physiological conditions via formation of $\mathrm{N}$ (4-methyl-5-oxo-1-imidazolin-2-yl)sarcosine (MG-HCr). Journal of Agricultural and Food Chemistry, 2015, 63(8): 2249-2256

8. Dornadula S, Elango B, Balashanmugam P, Palanisamy R, Kunka Mohanram R. Pathophysiological insights of methylglyoxal induced type-2 diabetes. Chemical Research in Toxicology, 2015, 28(9): $1666-1674$

9. Colzani M, De Maddis D, Casali G, Carini M, Vistoli G, Aldini G. Reactivity, selectivity, and reaction mechanisms of aminoguanidine, hydralazine, pyridoxamine, and carnosine as sequestering agents of 
reactive carbonyl species: a comparative study. ChemMedChem, 2016, 11(16): 1778-1789

10. Miyata T, van Ypersele de Strihou C, Kurokawa K, Baynes J W. Alterations in nonenzymatic biochemistry in uremia: origin and significance of "carbonyl stress" in long-term uremic complications. Kidney International, 1999, 55(2): 389-399

11. Aldini G, Dalle-Donne I, Facino R M, Milzani A, Carini M. Intervention strategies to inhibit protein carbonylation by lipoxidation-derived reactive carbonyls. Medicinal Research Reviews, 2007, 27(6): 817-868

12. Thornalley P J. Monosaccharide autoxidation in health and disease. Environmental Health Perspectives, 1985, 64: 297-307

13. Hayashi T, Namki M. Formation of two-carbon sugar fragment at an early stage of the browning reaction of sugar with amine. Agricultural and Biological Chemistry, 1980, 44(11): 2575-2580

14. Saadat D, Harrison D H. The crystal structure of methylglyoxal synthase from Escherichia coli. Structure, 1999, 7(3): 309-317

15. Phillips S A, Thornalley P J. The formation of methylglyoxal from triose phosphates. Investigation using a specific assay for methylglyoxal. European Journal of Biochemistry, 1993, 212(1): 101-105

16. Hopper D J, Cooper R A. The regulation of Escherichia coli methylglyoxal synthase: a new control site in glycolysis? FEBS Letters, 1971, 13(4): 213-216

17. Hopper D J, Cooper R A. The purification and properties of Escherichia coli methylglyoxal synthase. Biochemical Journal, 1972, 128(2): 321-329

18. Tötemeyer S, Booth N A, Nichols W W, Dunbar B, Booth I R. From famine to feast: the role of methylglyoxal production in Escherichia coli. Molecular Microbiology, 1998, 27(3): 553-562

19. Allaman I, Bélanger M, Magistretti P J. Methylglyoxal, the dark side of glycolysis. Frontiers in Neuroscience, 2015, 9: 23

20. Thornalley P J. Dicarbonyl intermediates in the maillard reaction. Annals of the New York Academy of Sciences, 2005, 1043(1): 111117

21. Angeloni C, Zambonin L, Hrelia S. Role of methylglyoxal in Alzheimer's disease. BioMed Research International, 2014, 2014 (2014): 238485

22. Klöpfer A, Spanneberg R, Glomb M A. Formation of arginine modifications in a model system of $N \alpha$-tert-butoxycarbonyl (Boc)arginine with methylglyoxal. Journal of Agricultural and Food Chemistry, 2011, 59(1): 394-401

23. Vistoli G, De Maddis D, Cipak A, Zarkovic N, Carini M, Aldini G. Advanced glycoxidation and lipoxidation end products (AGEs and ALEs): an overview of their mechanisms of formation. Free Radical Research, 2013, 47(S1): 3-27

24. Oya T, Hattori N, Mizuno Y, Miyata S, Maeda S, Osawa T, Uchida K. Methylglyoxal modification of protein. Chemical and immunochemical characterization of methylglyoxal-arginine adducts. Journal of Biological Chemistry, 1999, 274(26): 18492-18502

25. Shipanova I N, Glomb M A, Nagaraj R H. Protein modification by methylglyoxal: chemical nature and synthetic mechanism of a major fluorescent adduct. Archives of Biochemistry and Biophysics, 1997, 344(1): 29-36

26. Ahmed M U, Brinkmann Frye E, Degenhardt T P, Thorpe S R, Baynes J W. NE-(Carboxyethyl)lysine, a product of the chemical modification of proteins by methylglyoxal, increases with age in human lens proteins. Biochemical Journal, 1997, 324(2): 565-570

27. Nasiri R, Field M J, Zahedi M, Moosavi-Movahedi A A. Crosslinking mechanisms of arginine and lysine with $\alpha, \beta$-dicarbonyl compounds in aqueous solution. Journal of Physical Chemistry A, 2011, 115(46): 13542-13555

28. Nagaraj R H, Shipanova N, Faust F M. Protein cross-linking by the Maillard reaction isolation, characterization, and in vivo detection of a lysine-lysine cross-link derived from methylglyoxal. Journal of Biological Chemistry, 196, 271(32): 19338-19345

29. Lo T W, Westwood M E, McLellan A C, Selwood T, Thornalley P J. Binding and modification of proteins by methylglyoxal under physiological conditions. A kinetic and mechanistic study with $N$ alpha-acetylarginine, $N$ alpha-acetylcysteine, and $N$ alpha-acetyllysine, and bovine serum albumin. Journal of Biological Chemistry, 1994, 269(51): 32299-32305

30. Nemet I, Varga-Defterdarović L. Methylglyoxal-derived $\beta$-carbolines formed from tryptophan and its derivates in the Maillard reaction. Amino Acids, 2007, 32(2): 291-293

31. Ramasamy R, Yan S F, Schmidt A M. Methylglyoxal comes of AGE. Cell, 2006, 124(2): 258-260

32. de Arriba S G, Stuchbury G, Yarin J, Burnell J, Loske C, Münch G. Methylglyoxal impairs glucose metabolism and leads to energy depletion in neuronal cells-protection by carbonyl scavengers. Neurobiology of Aging, 2007, 28(7): 1044-1050

33. Kaufmann E, Boehm B O, Süssmuth S D, Kientsch-Engel R, Sperfeld A, Ludolph A C, Tumani H. The advanced glycation endproduct $\mathrm{N}$ epsilon-(carboxymethyl)lysine level is elevated in cerebrospinal fluid of patients with amyotrophic lateral sclerosis. Neuroscience Letters, 2004, 371(2-3): 226-229

34. Southern L, Williams J, Esiri M M. Immunohistochemical study of $\mathrm{N}$-epsilon-carboxymethyl lysine (CML) in human brain: relation to vascular dementia. BMC Neurology, 2007, 7(1): 35

35. Matafome P, Sena C, Seiça R. Methylglyoxal, obesity, and diabetes. Endocrine, 2013, 43(3): 472-484

36. Sena C M, Matafome P, Crisóstomo J, Rodrigues L, Fernandes R, Pereira P, Seiça R M. Methylglyoxal promotes oxidative stress and endothelial dysfunction. Pharmacological Research, 2012, 65(5): 497-506

37. Chang T, Wang R, Wu L. Methylglyoxal-induced nitric oxide and peroxynitrite production in vascular smooth muscle cells. Free Radical Biology \& Medicine, 2005, 38(2): 286-293

38. Dhar A, Desai K, Kazachmov M, Yu P, Wu L. Methylglyoxal production in vascular smooth muscle cells from different metabolic precursors. Metabolism: Clinical and Experimental, 2008, 57(9): $1211-1220$

39. Ward R A, McLeish K R. Methylglyoxal: a stimulus to neutrophil oxygen radical production in chronic renal failure? Nephrology, Dialysis, Transplantation, 2004, 19(7): 1702-1707

40. Kalapos M P, Littauer A, de Groot H. Has reactive oxygen a role in methylglyoxal toxicity? A study on cultured rat hepatocytes. Archives of Toxicology, 1993, 67(5): 369-372

41. Amicarelli F, Colafarina S, Cattani F, Cimini A, Di Ilio C, Ceru M P, Miranda M. Scavenging system efficiency is crucial for cell resistance to ROS-mediated methylglyoxal injury. Free Radical Biology \& Medicine, 2003, 35(8): 856-871

42. Paget C, Lecomte M, Ruggiero D, Wiernsperger N, Lagarde M. 
Modification of enzymatic antioxidants in retinal microvascular cells by glucose or advanced glycation end products. Free Radical Biology \& Medicine, 1998, 25(1): 121-129

43. Odani H, Shinzato T, Matsumoto Y, Usami J, Maeda K. Increase in three $\alpha, \beta$-dicarbonyl compound levels in human uremic plasma: specific in vivo determination of intermediates in advanced Maillard reaction. Biochemical and Biophysical Research Communications, 1999, 256(1): 89-93

44. Jia X, Olson D J, Ross A R, Wu L. Structural and functional changes in human insulin induced by methylglyoxal. FASEB Journal, 2006, 20(9): 1555-1557

45. Oliveira L M, Lages A, Gomes R A, Neves H, Família C, Coelho A $\mathrm{V}$, Quintas A. Insulin glycation by methylglyoxal results in nativelike aggregation and inhibition of fibril formation. BMC Biochemistry, 2011, 12(1): 41

46. Tóth A E, Tóth A, Walter F R, Kiss L, Veszelka S, Ózsvári B, Puskás L G, Heimesaat M M, Dohgu S, Kataoka Y, Rákhely G, Deli M A. Compounds blocking methylglyoxal-induced protein modification and brain endothelial injury. Archives of Medical Research, 2014, 45 (8): 753-764

47. Kuhla B, Lüth H J, Haferburg D, Boeck K, Arendt T, Münch G. Methylglyoxal, glyoxal, and their detoxification in Alzheimer's disease. Annals of the New York Academy of Sciences, 2005, 1043 (1): 211-216

48. Münch G, Westcott B, Menini T, Gugliucci A. Advanced glycation endproducts and their pathogenic roles in neurological disorders. Amino Acids, 42(4): 1221-1236

49. Vitek M P, Bhattacharya K, Glendening J M, Stopa E, Vlassara H, Bucala R, Manogue K, Cerami A. Advanced glycation end products contribute to amyloidosis in Alzheimer disease. Proceedings of the National Academy of Sciences of the United States of America, 1994, 91(11): 4766-4770

50. Naudí A, Jové M, Cacabelos D, Ayala V, Cabre R, Caro P, Gomez J, Portero-Otín M, Barja G, Pamplona R. Formation of S-(carboxymethyl)-cysteine in rat liver mitochondrial proteins: effects of caloric and methionine restriction. Amino Acids, 2013, 44(2): 361371

51. Negre-Salvayre A, Salvayre R, Augé N, Pamplona R, Portero-Otín M. Hyperglycemia and glycation in diabetic complications. Antioxidants \& Redox Signalling, 2009, 11(12): 3071-3109

52. Takamiya R, Takahashi M, Myint T, Park Y S, Miyazawa N, Endo T, Fujiwara N, Sakiyama H, Misonou Y, Miyamoto Y, Fujii J, Taniguchi N. Glycation proceeds faster in mutated $\mathrm{Cu}, \mathrm{Zn}$ superoxide dismutases related to familial amyotrophic lateral sclerosis. FASEB Journal, 2003, 17(8): 938-940

53. Kikuchi S, Shinpo K, Ogata A, Tsuji S, Takeuchi M, Makita Z, Tashiro K. Detection of N epsilon-(carboxymethyl)lysine (CML) and non-CML advanced glycation end-products in the anterior horn of amyotrophic lateral sclerosis spinal cord. Amyotrophic Lateral Sclerosis and Other Motor Neuron Disorders, 2002, 3(2):
63-68

54. Kalousová M, Zima T, Tesař V, Dusilová-Sulková S, Škrha J. Advanced glycoxidation end products in chronic diseases-clinical chemistry and genetic background. Mutation Research, 2005, 579 (1-2): 37-46 doi:10.1016/j.mrfmmm.2005.03.024

55. Andersson A, Covacu R, Sunnemark D, Danilov A I, Dal Bianco A, Khademi M, Wallström E, Lobell A, Brundin L, Lassmann H, Harris R A. Pivotal advance: HMGB1 expression in active lesions of human and experimental multiple sclerosis. Journal of Leukocyte Biology, 2008, 84(5): 1248-1255

56. Münch G, Lüth H J, Wong A, Arendt T, Hirsch E, Ravid R, Riederer P. Crosslinking of $\alpha$-synuclein by advanced glycation endproductsan early pathophysiological step in Lewy body formation? Journal of Chemical Neuroanatomy, 2000, 20(3-4): 253-257

57. Dalfó E, Portero-Otín M, Ayala V, Martínez A, Pamplona R, Ferrer I. Evidence of oxidative stress in the neocortex in incidental Lewy body disease. Journal of Neuropathology and Experimental Neurology, 2005, 64(9): 816-830

58. Dukic-Stefanovic S, Schinzel R, Riederer P, Münch G. AGES in brain ageing: AGE-inhibitors as neuroprotective and anti-dementia drugs? Biogerontology, 2001, 2(1): 19-34

59. Wu C H, Yen G C. Inhibitory effect of naturally occurring flavonoids on the formation of advanced glycation endproducts. Journal of Agricultural and Food Chemistry, 2005, 53(8): 31673173

60. Sang S, Shao X, Bai N, Lo C Y, Yang C S, Ho C T. Tea polyphenol (-)-epigallocatechin-3-gallate: a new trapping agent of reactive dicarbonyl species. Chemical Research in Toxicology, 2007, 20(12): 1862-1870

61. Lv L, Shao X, Chen H, Ho C T, Sang S. Genistein inhibits advanced glycation end product formation by trapping methylglyoxal. Chemical Research in Toxicology, 2011, 24(4): 579-586

62. Li X, Zheng T, Sang S, Lv L. Quercetin inhibits advanced glycation end product formation by trapping methylglyoxal and glyoxal. Journal of Agricultural and Food Chemistry, 2014, 62(50): 1215212158

63. Shao X, Bai N, He K, Ho C T, Yang C S, Sang S. Apple polyphenols, phloretin and phloridzin: new trapping agents of reactive dicarbonyl species. Chemical Research in Toxicology, 2008, 21(10): 2042-2050

64. Wang P, Chen H, Sang S. Trapping methylglyoxal by genistein and its metabolites in mice. Chemical Research in Toxicology, 2016, 29 (3): 406-414

65. Lo C Y, Hsiao W T, Chen X Y. Efficiency of trapping methylglyoxal by phenols and phenolic acids. Journal of Food Science, 2011, 76(3): H90-H96

66. Shao X, Chen H, Zhu Y, Sedighi R, Ho C T, Sang S. Essential structural requirements and additive effects for flavonoids to scavenge methylglyoxal. Journal of Agricultural and Food Chemistry, 2014, 62(14): 3202-3210 